\title{
Cerâmicas Nacionais com Alta Permissividade na Fabricação de Filtros em Freqüência de Microondas
}

\author{
F. R. Borges, J. A. Atizani, M. O. Moraes, H. E. Hernández-Figueroa \\ Departamento de Microondas e Óptica, Faculdade de Engenharia Elétrica e de Computação, Universidade Estadual de Campinas (UNICAMP), Brasil \\ Av. Albert Einstein, 400, CEP 13083-852
}

\begin{abstract}
Resumo - A produção emergente de cerâmicas dielétricas no país permite o desenvolvimento de dispositivos na faixa de microondas que têm aplicações práticas diversas, tais como filtros e antenas dielétricas de dimensões reduzidas. Neste trabalho tem-se a análise de uma cavidade ressonante e sua imediata extensão para o projeto de um filtro dielétrico usando cerâmicas com alta permissividade, o que permite a construção de um dispositivo mais leve e compacto do que os similares desenvolvidos usando cerâmicas comercialmente disponíveis atualmente.
\end{abstract}

Palavras-chaves - Filtro dielétrico, Ressoador dielétrico, Simulação eletromagnética.

\begin{abstract}
The emerging production of dielectric ceramics in the country allows for the development of microwave devices that have practical applications, such as filters and dielectric antennas of small dimensions. This work focus in the analysis of a resonant cavity and its immediate extension to the project of a dielectric filter using ceramics with high permitivity, which allows the construction of a device lighter and more compact than similar filters using ceramics commercially available today .
\end{abstract}

Keywords — Dielectric filter, Dielectric resonator, Electromagnetic simulation.

\section{INTRODUÇÃO}

A pesquisa e o desenvolvimento de dispositivos de microondas, como filtros e antenas, é de vital importância para a economia do país. Com a recente proibição da importação de alguns tipos de cerâmicas dielétricas por parte dos Estados Unidos, em particular aquelas para aplicações militares, a necessidade de produzi-las no Brasil torna-se de extrema importância.

O grupo coordenado pelo professor Dr. Sérgio Sombra, da Universidade Federal do Ceará, UFC, está desenvolvendo técnicas para produzir cerâmicas dielétricas com permissividade relativa entre 50 e 150 [1] - [3], o que torna possível não apenas desenvolver nacionalmente, mas também miniaturizar dispositivos de microondas projetados usando as mesmas, pois se encontra, comercialmente, cerâmicas com permissividade relativa até a ordem de 80 .

Este trabalho apresenta a construção de um filtro com ressoadores dielétricos cuja permissividade relativa é avaliada considerando os efeitos da estrutura que envolve as mesmas.

F. R. Borges, fabiano@dmo.fee.unicamp.br, J. A. Atizani, juatizani@fee.unicamp.br, M. O. Moraes,ra03495@fee.unicamp.br, H. E. Hernández-Figueroa, hugo@dmo.fee.unicamp.br, Tel +55-19-3521-3720, Fax +5519-3289-1395

Este trabalho foi financiado pelo CAPES.
Inicialmente, tem-se a definição do tipo de dispositivo bem como do modo mais adequado para alimentar o circuito, uma etapa fundamental do projeto. A seguir, resultados de simulação computacional, obtidos com o software CST Design Studio ${ }^{\mathrm{TM}}$ [4], são comparados com medições realizadas em um protótipo construído a fim de validar as mesmas. Por fim, tem-se o exemplo de um segundo dispositivo de microondas projetado usando as cerâmicas, um filtro passa-banda de segunda ordem.

\section{DisPositivos PROJETADOS}

\section{A. Cavidade ressonante}

A primeira etapa para projetar o dispositivo é determinar a forma e a freqüência de ressonância do ressoador levando em consideração as interferências dos elementos que compõem o dispositivo. Isto exige o conhecimento da distribuição dos campos elétrico e magnético dentro do ressoador e da maneira correta de excitá-lo.

1) Distribuição de campos dentro de um ressoador: Optouse por ressoadores de formato cilíndrico, por serem os mais usados como estruturas cerâmicas ressonantes na construção de filtros. Se a altura do cilindro for maior que seu diâmetro, tem-se como modo fundamental o $T M_{01 \delta}$, cuja distribuição de campos é mostrada na Fig. 1, enquanto a Fig. 2 mostra o modo fundamental $T E_{01 \delta}$, que é o modo dominante se a altura for menor que o diâmetro do cilindro [5].

2) Excitação dos modos de um ressoador cilíndrico: A maneira mais simples de excitar o modo $T M_{01 \delta}$ é colocar um condutor, como a ponta de um cabo coaxial ou uma trilha de microfita, alinhado com o eixo $z$ conforme indicado na Fig. 1. Se a freqüência injetada permitir a excitação do modo dominante, surge um acoplamento magnético entre o alimentador e o ressoador, como é possível perceber pela Fig. 1b, e o campo elétrico segue a distribuição indicado na Fig. 1a.

Para excitar o modo $T E_{01 \delta}$, basta colocar um condutor alinhado com o eixo y indicado na Fig. 2. Se a freqüência injetada permitir a excitação do modo dominante, surgirá um acoplamento magnético entre o alimentador e o ressoador, como é possível perceber pela Fig. 2b, e o campo elétrico segue a distribuição indicado na Fig. 2a.

3) Determinação da freqüência de ressonância do conjunto: A determinação da freqüência de ressonância do conjunto pode ser feita com grande precisão a partir de um modelo de 
simulação bastante simplificado em relação ao modelo completo da estrutura. O modelo simplificado deve ser composto apenas de uma cavidade e de um ressoador, como mostra a Fig. 3, em oposição ao modelo completo, que tem ainda um substrato, um coaxial e uma trilha de microfita, mostrados na Fig.4. Além disso, as dimensões do coaxial e da trilha e sua posição em relação ao ressoador devem ser ajustadas.

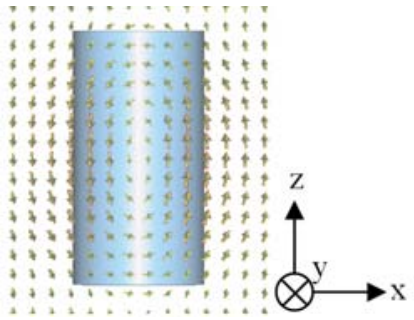

(a)

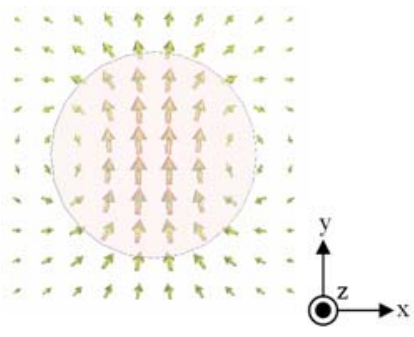

(b)
Fig. 1. Representação da distribuição de campo para o modo $T M_{01 \delta}$. (a) Campo elétrico. (b) Campo magnético.

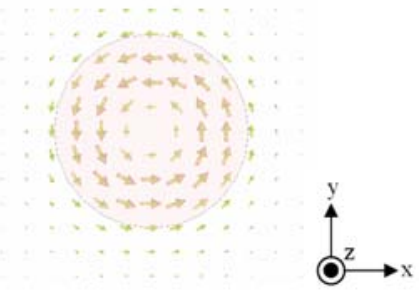

(a)

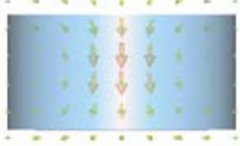

(b)

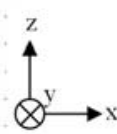

Fig. 2. Representação da distribuição de campo para o modo $T E_{01 \delta}$.

(a) Campo elétrico. (b) Campo magnético.

No modelo simplificado as dimensões internas $a, b$ e $c$ da cavidade ressonante, juntamente com as dimensões $h$ e $d$ do ressoador e sua permissividade, e a distância $t$ da base do ressoador em relação ao fundo da caixa, definem a freqüência de ressonância do conjunto. O substrato usado apresenta uma permissividade relativa da ordem de 4,5, um valor pequeno quando comparado à permissividade do ressoador, de modo que sua influência na ressonância pode ser desprezada. Entretanto, sua espessura e sua posição em relação ao fundo da cavidade, cuja soma é igual a $t$, devem ser levados em conta no cômputo da freqüência de ressonância do conjunto. A diferença entre a freqüência obtida com este modelo e com o modelo completo é da ordem de $1 \%$, e o tempo de simulação envolvido é consideravelmente menor.

A partir deste modelo são obtidos os autovalores simulados sem a necessidade de uma fonte externa para a excitação do conjunto. Isto elimina a necessidade do projeto da alimentação neste estágio do projeto. O que garante o surgimento do modo correto é o uso das condições de contorno apropriadas, bem como a razão altura/diâmetro do ressoador [5].

4) Condições de contorno: Em virtude das características do software usado, para garantir o surgimento do modo dominante e não um modo da própria cavidade é importante usar condições de simetria apropriadas. Por inspeção da dis- tribuição dos campos para o modo $T E_{01 \delta}$, três condições de simetria podem ser usadas (veja a Fig. 2): Perfectly Electrical Condutctor (PEC), ao longo dos planos $x z$ e $y z$, pois o campo elétrico é normal a estes planos e, de modo totalmente dual, o campo magnético é tangente a estes planos, e um Perfectly Magnetic Conductor (PMC), ao longo do plano $x y$, uma vez que o campo magnético é normal a este plano e, por dualidade, o campo elétrico é tangente ao mesmo. Esta última condição somente pode ser aplicada se o ressoador for posicionado no centro geométrico da caixa. De forma similar, pela distribuição dos campos para o modo $T M_{01 \delta}$, podem-se usar duas condições de simetria (veja a Fig. 1): PEC ao longo do plano $y z$ e um PMC ao longo do plano $x y$. Novamente, esta última condição somente pode ser usada se o ressoador for colocado bem no centro da caixa em relação aos três eixos ortogonais.

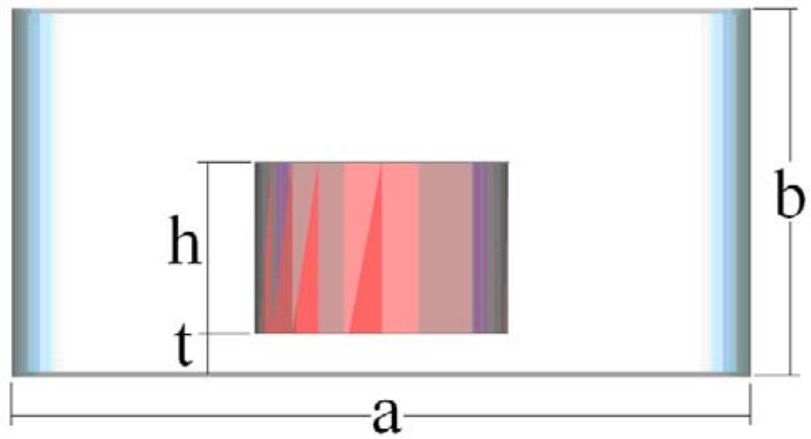

(a)

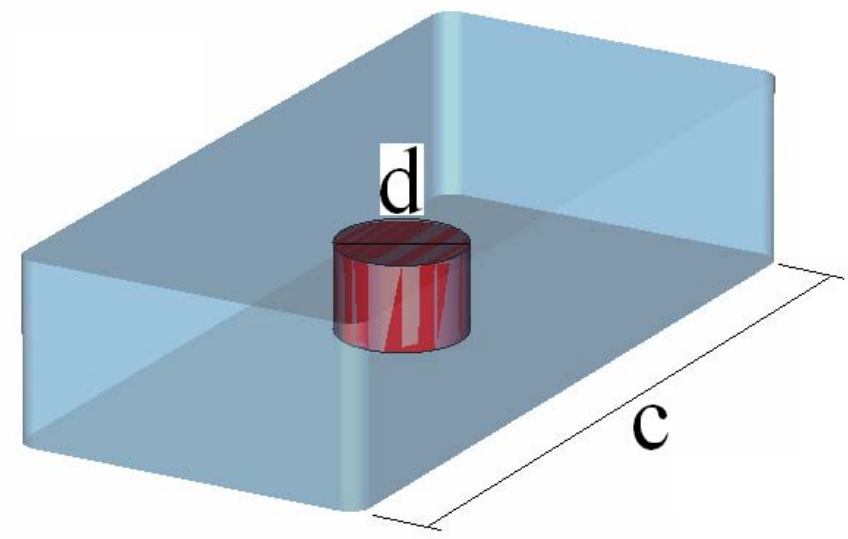

(b)

Fig. 3. Modelo simplificado para determinação da freqüência de ressonância do conjunto. (a) Vista de frente. (b) Visão tridimensional.

5) Modelo Completo: Na Fig. 4 é apresentado o modelo esquemático da estrutura com um ressoador cilíndrico.

O dispositivo completo como foi construído é composto por uma estrutura de alumínio como uma cavidade ressonante, um substrato de fibra de vidro como base de apoio para o ressoador cilíndrico e linhas de microfitas para excitar o ressoador e receber o sinal na saída. Quatro parafusos foram colocados por baixo do substrato para posicioná-lo em uma altura ajustável a partir do fundo da cavidade. 
Usou-se uma linha de microfita para excitar o modo $T E_{01 \delta}$ no conjunto, o que requer que usemos um ressoador com altura menor que o diâmetro.

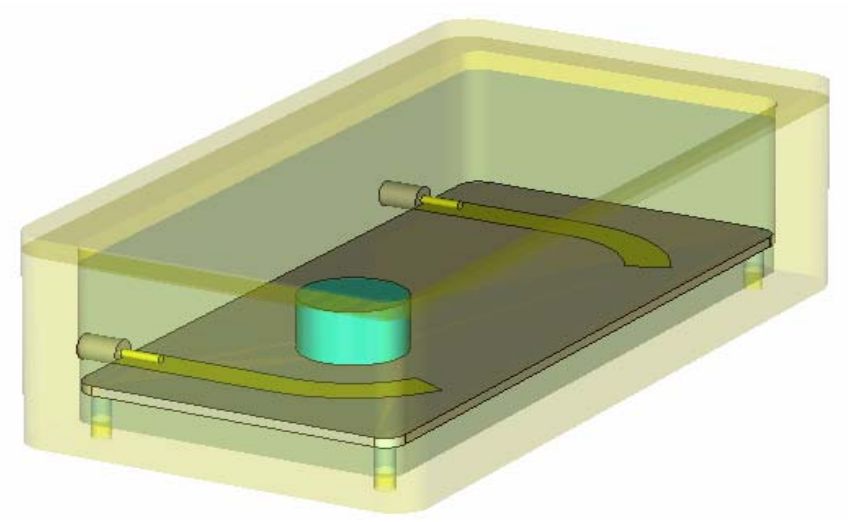

Fig. 4. Modelo completo da estrutura para teste.

Como a profundidade pelicular do alumínio na faixa de freqüência de microondas é da ordem de micrometros, a espessura das paredes da cavidade pode ser feita a mais fina possível. Isto significa que a dissipação da energia que não é acoplada no ressoador e que atinge as paredes da cavidade ocorre em apenas alguns micrometros. Contudo, a tolerância mecânica do processo de fabricação exige que a espessura da caixa seja da ordem de milímetros.

Após inserir o substrato e ajustar o comprimento da linha de microfita para um valor da ordem de um quarto de comprimento de onda guiado, obtemos uma cavidade ressonante capaz de excitar o modo $T M_{01 \delta}$ no ressoador que colocarmos no interior da mesma.

\section{B. Filtro}

1) Características gerais: Filtros são dispositivos de duas portas caracterizados matematicamente por uma função de transferência do tipo [6]

$$
\left|S_{21}(j \omega)\right|^{2}=\frac{1}{1+q^{2}\left[F_{n}(\omega)\right]^{2}}
$$

onde $q$ está relacionado com o ripple na banda passante e $F_{n}(j \omega)$ é uma função que depende do tipo de filtro escolhido. Para filtros Butterworth, têm-se [4]

$$
\begin{gathered}
q=1 \\
F_{n}(\omega)=\frac{\omega}{\omega_{c}}
\end{gathered}
$$

onde $\omega_{c}$ é a freqüência de corte do filtro. Este tipo de filtro não apresenta ripple na banda passante $(q=1)$. Para os filtros Chebyshev, têm-se [6]

$$
\begin{aligned}
A_{p} & =10 \log \left(1+q^{2}\right) \\
F_{n}(\omega) & =\left\{\begin{array}{l}
1 \\
\omega \\
2 \omega F_{n-1}(\omega)-F_{n-2}(\omega)
\end{array}\right.
\end{aligned}
$$

onde $A_{p}$ é a atenuação na banda passante definida em decibéis. Como para este filtro $q \neq 1$, o mesmo apresenta ripple na banda passante, porém, tem uma atenuação maior fora da faixa de operação quando comparado ao filtro Butterworth. Isto significa que o filtro Chebyshev é mais seletivo, e esta é a razão principal para que ele seja um dos filtros mais utilizados.

Para projetar um filtro Chebyshev deve-se definir a freqüência central $f_{0}$, o atenuação $A_{p}$ na banda passante, a freqüência de corte $\omega_{c}$ nos limites do ripple, o nível de rejeição $A_{s}$ e as freqüências limite $\omega_{s}$ na banda de rejeição.

2) Projeto de um filtro usando elementos de parâmetros concentrados: A partir das especificações iniciais, os passos para obter os elementos da rede que sintetiza a função de transferência definida por (1) são [6]:

- Projeta-se um filtro passa-baixa normalizado por $\omega_{c}=1$ e centrado em $\omega_{0}=0$.

- A função de transferência resultante pode ser convertida em uma rede de indutores/capacitores usando técnicas de síntese de circuito. No caso dos filtros Butterworth e Chebyshev, há soluções analíticas que mapeiam a função de transferência passa-baixas diretamente em elementos $L$ e $C$, o que simplifica esta passagem.

- A partir de uma transformação simples de freqüência, converte-se o circuito passa-baixas normalizado em um circuito passa-banda que satisfaça (1) de acordo com os requisitos do filtro.

O problema desta abordagem é que, em microondas, os indutores e capacitores a parâmetros concentrados obtidos não podem ser usados porque sofrem efeitos parasitas. Para contornar este problema, é necessário converter o circuito obtido em um circuito equivalente de parâmetros distribuídos [7].

3) Conversão de um filtro de parâmetros concentrados para um filtro de parâmetros distribuídos: A principal ferramenta por trás desta conversão é o inversor de impedância, que pode ser, por exemplo, uma linha com um quarto de comprimento de onda. A função deste elemento é a de inverter a impedância de saída de modo que

$$
Z_{\text {in }}=\frac{k}{Z_{\text {out }}}
$$

onde $Z_{\text {in }}$ é a impedância vista na entrada do elemento, $Z_{\text {out }}$ é a impedância na saída do mesmo e $k$ é uma constante. No caso de uma linha de microfita, $k=Z_{0}^{2}$, a impedância característica da mesma. Há, também, um quadripolo formado por indutores e capacitores [7] que desempenha a mesma função, no qual os elementos têm valores igual a

$$
\begin{aligned}
& k=\frac{1}{\omega C} \\
& k=\omega L
\end{aligned}
$$

onde $L$ é uma indutância e $C$ é uma capacitância que compõem o mesmo. Existem duas vantagens fundamentais nesta operação [6,7]: 
- $\quad$ a rede de elementos $L C$ paralelo e série é convertida em uma única rede, toda em série ou toda em paralelo, com a vantagem adicional de que todos os seus componentes são exatamente iguais. Esta vantagem adicional vem do fato de que se os elementos $L C$ são todos iguais, os elementos distribuídos que os representarão no circuito final (linhas de microfita, ressoadores cilíndricos etc.) serão todos iguais, isto é, têm as mesmas dimensões e características, o que facilita a construção.

- o inversor de impedância pode ser substituído por um acoplamento elétrico ou magnético, favorecendo o uso de elementos distribuídos.

Deve-se observar que o uso do inversor de impedância implica em limitar a banda de operação do filtro, visto que o inversor possui uma banda limitada, pois, idealmente, apenas em uma freqüência a operação de inversão é realizada corretamente. Isto torna esta técnica útil para a construção de filtros de faixa estreita [7].

\section{RESULTADOS}

Baseado nos conceitos descritos anteriormente, e em simulações usando o software CST, foi construído um protótipo da cavidade ressonante usando alumínio e um substrato do tipo FR-4, com permissividade relativa estimada em 4,5. As linhas de microfita para alimentar os ressoadores foram projetadas para uma impedância característica de $50 \Omega$. Pelo fato de o dispositivo operar em microondas, usou-se conectores do tipo SMA para alimentar externamente o conjunto. A Fig. 5 mostra o protótipo construído com base nos dados obtidos via simulação eletromagnética.

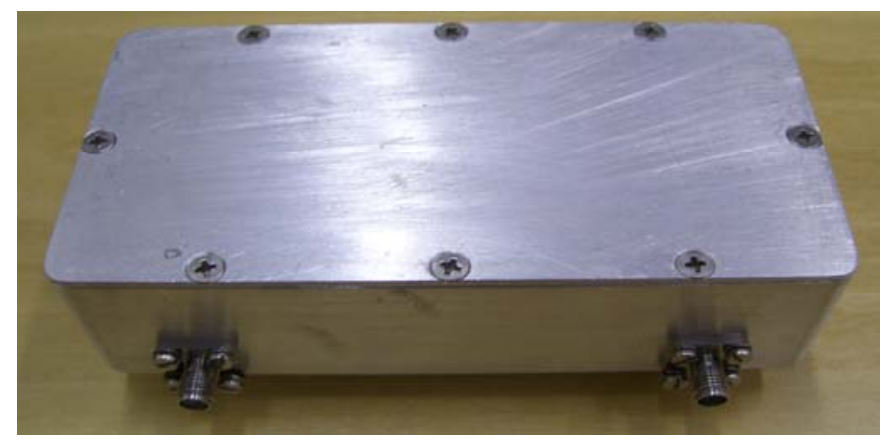

(a)

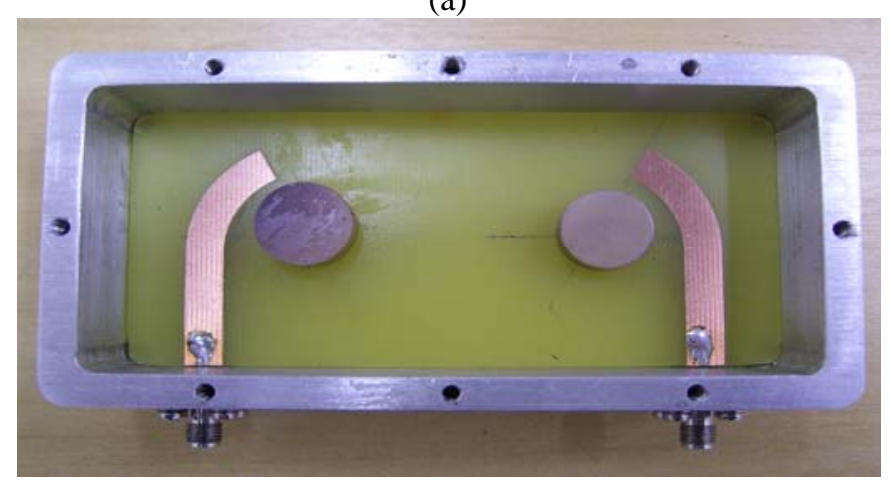

(b)

Fig. 5. Cavidade ressonante fabricada. (a) Imagem com a tampa fechada. (b) Visão interna.
A Tabela I mostra as características das cerâmicas usadas nos testes de laboratório e nas simulações, de acordo com dados fornecidos pelo fabricante.

TABELA I. CARACTERÍSTICAS DAS CERÂMICAS FABRICADAS.

\begin{tabular}{cccc}
\hline Amostra & $\varepsilon_{\mathrm{r}}$ & $\begin{array}{c}\text { Tangente de } \\
\text { Perdas }\end{array}$ & $\begin{array}{c}\text { Freqüência das Medições } \\
(\mathrm{MHz})\end{array}$ \\
\hline 1 & 91 & $3,1.10^{-3}$ & 3597 \\
2 & 141 & $1,7.10^{-3}$ & 2050 \\
3 & 155 & $4,9.10^{-3}$ & 2603 \\
\hline
\end{tabular}

Usou-se o parâmetro $S_{11}$ para avaliar a freqüência de ressonância do dispositivo. As Figs. 6 a 8 mostram uma comparação entre a ressonância simulada e medida usando os dados indicados Tabela I e de acordo com o setup mostrado na Fig. 4.

A Tabela II mostra a diferença entre a freqüência de ressonância simulada e a medida para cada um dos ressoadores avalidos.

TABELA II. ERRO PERCENTUAL DA FREQÜÊNCIA DE RESSONÂNCIA.

\begin{tabular}{cc}
\hline$\varepsilon_{\mathrm{r}}$ & Erro na Freqüência de Ressonância (\%) \\
\hline 91 & 1,5 \\
141 & 1,3 \\
155 & 1,2 \\
\hline
\end{tabular}

A diferença média entre a freqüência de ressonância projetada e a medida é da ordem de 1,3\% . Esta diferença certamente deve-se ao fato de que os métodos de medição são diferentes, e que as medições feitas pelo grupo do prof. Dr. Sérgio Sombra foram realizadas em freqüências diferentes e acima da freqüência em que as cerâmicas foram avaliadas neste trabalho. Enquanto o grupo do prof. Dr. Sérgio Sombra usa o ressoador como uma antena exposta ao ambiente, apresentando, portanto, perdas por radiação, o modelo aqui apresentado não leva em conta o ambiente externo, pois a cavidade metálica o isola do conjunto.

$\mathrm{O}$ fato de todas as curvas simuladas mostrarem um valor diferente, porém sempre menor que aquele informado pelo fabricante indica uma tendência clara de que ambos os métodos de medição levam a resultados consistentes.

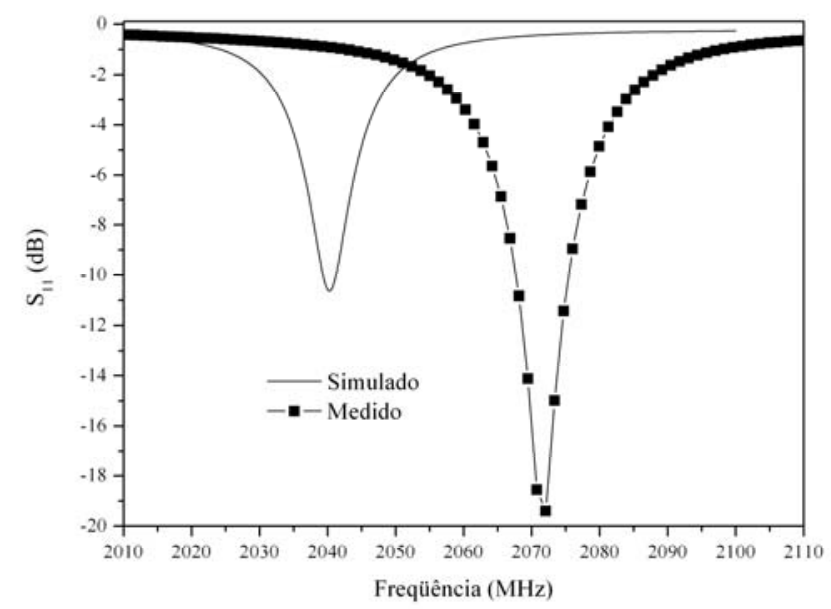

Fig. 6. Ressonância do conjunto para $\varepsilon_{r}=91$. 


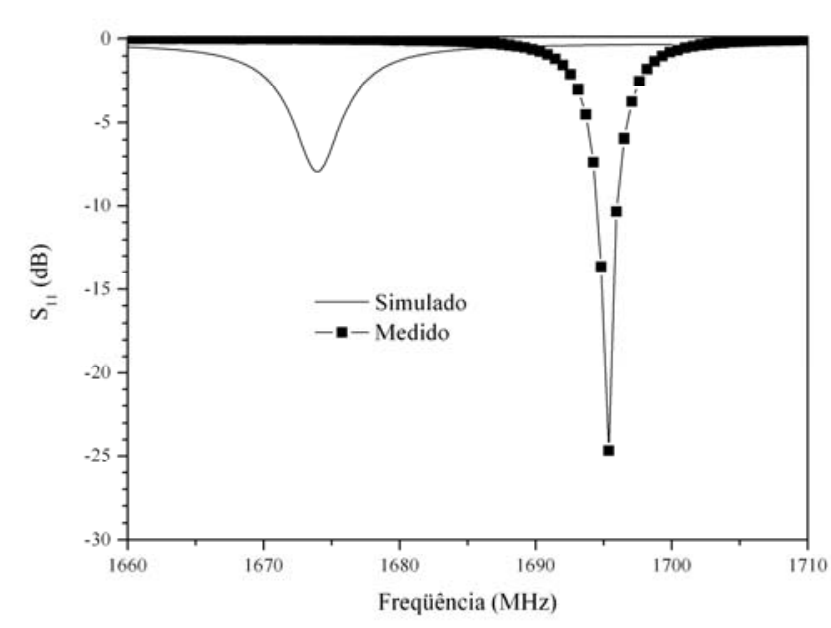

Fig. 7. Ressonância do conjunto para $\varepsilon_{r}=141$.

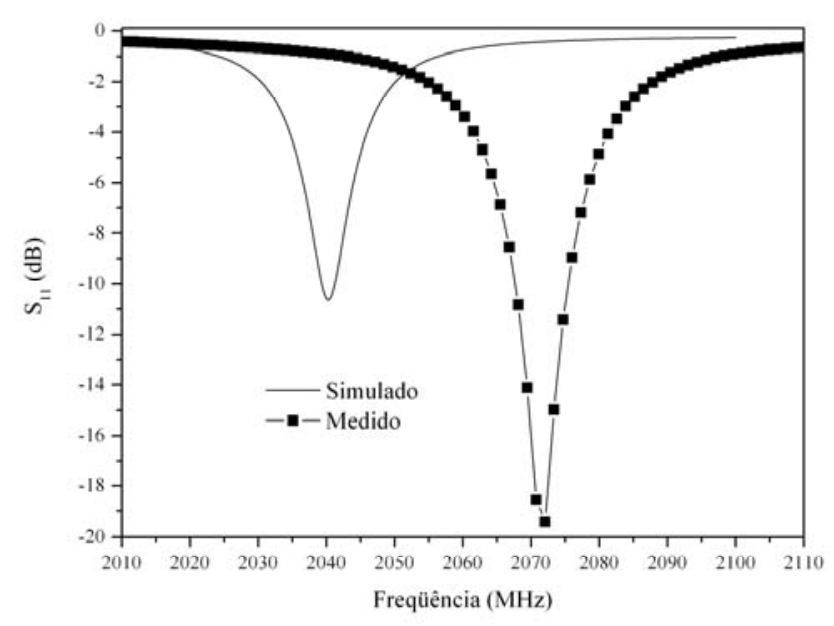

Fig. 8. Ressonância do conjunto para $\varepsilon_{r}=155$.

Se considerarmos que a diferença entre as freqüências medida e simulada deve-se somente ao valor da permissividade relativa, podemos fazer uma simulação mudando apenas o valor da permissividade até que a freqüência de ressonância das curvas simulada e medida seja a mesma. Fazendo isso, obtemos uma variação média a ordem de $2,5 \%$, um valor bastante reduzido dadas as diferenças nos métodos de medição.

A partir destes resultados e da teoria de filtros, projetou-se um filtro dielétrico de segunda ordem. A Fig. 10 mostra a resposta em freqüência do filtro com dois ressoadores dielétricos para o caso ideal, sem perdas, e para os casos com tangente de perda dos ressoadores entre $10^{-3}$ e $10^{-4}$, além da função de transferência teórica obtida a partir das especificações do filtro e usando (1) e (3). Os resultados foram obtidos via simulação usando o software CST Design Studio ${ }^{\mathrm{TM}}$.

A perda total para os três casos simulados está indicada na Tabela III.

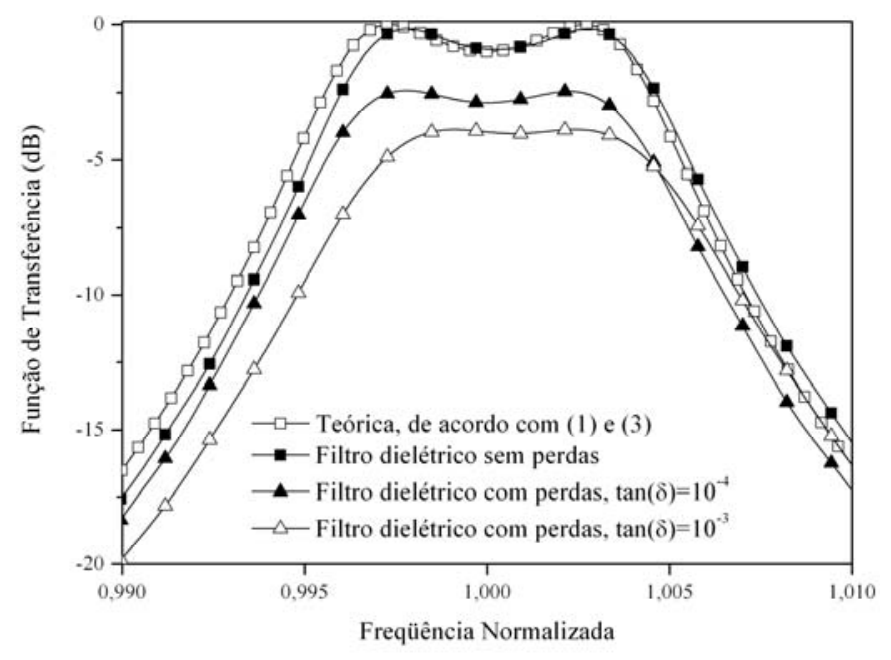

Fig. 10. Resposta em freqüência do filtro para $\varepsilon_{\mathrm{r}}=100$.

TABELA III. Características das cerâmicas fabricadas.

\begin{tabular}{ccc}
\hline $\begin{array}{c}\text { Tangente } \\
\text { de Perdas }\end{array}$ & Perdas no Ressoador $(\mathrm{dB})$ & Perda de Inserção do Filtro (dB) \\
\hline $10^{-3}$ & $-1,90$ & $-3,90$ \\
$10^{-4}$ & $-0,20$ & $-2,40$ \\
\hline
\end{tabular}

As curvas teórica e simulada do filtro dielétrico desconsiderando-se as perdas mostram uma excelente concordância de valores. É importante ressaltar que a curva teórica foi obtida diretamente de uma função de transferência do tipo (1) (3), enquanto que a curva simulada foi obtida usando o modelo baseado na Fig. 4. A inserção de outras perdas no conjunto - caixa de alumínio, substrato de FR-4, trilhas de cobre, coaxial e ressoadores - mostram uma perda de inserção que varia 40\% para uma variação de 10 vezes na tangente de perdas. Um modo de minimizar isso seria reduzir a extensão do substrato, que passaria a dissipar menos energia, uma vez que a área seria reduzida. Outra possibilidade de reduzir perdas na cavidade é aplicar uma camada de prata, com a espessura da profundidade pelicular do alumínio dentro da cavidade, seguida de uma camada fina de ouro na parte interna da cavidade, a fim de evitar a oxidação da prata. Sendo a prata um condutor melhor que o alumínio, menos energia seria dissipada no metal, e a energia consumida pela cavidade seria reduzida, diminuindo assim a perda de inserção.

O protótipo para obter as curvas mostradas na Fig. 10 está em fase de construção.

\section{CONCLUSÕES}

Os resultados preliminares usando um ressoador mostram uma boa concordância com a resposta obtida via simulação, como mostram as Figs. 6 a 9, o que indica a viabilidade de fabricação de um filtro de microondas de faixa estreita usando ressoadores dielétricos de alta permissividade,possibilitando assim miniaturizar filtros usados comercialmente, uma vez que estes usam permissividade relativa da ordem de 80 , e o grupo do prof. Dr. Sérgio Sombra obtém permissividade relativa de até 150 . 


\section{AGRADECIMENTOS}

Os autores agradecem aos senhores Alexandre M. P. A. da Silva, Daniel N. Duplat e Luciano P. Oliveira, da Unicamp, pelas discussões técnicas desenvolvidas ao longo deste projeto.

\section{REFERENNCIAS}

[1] A. F. L. Almeida, P. B. A. Fechini, J. C. Goes, M. A. Valente, M. A. R. Miranda, A. S. B. Sombra, "Dielectric properties of $\mathrm{BaTiO}_{3}$ (BTO)$\mathrm{CaCu}_{3} \mathrm{Ti}_{4} \mathrm{O}_{12}$ (CCTO) composite screen-printed thick films for high dielectric constant devices in the medium frequency (MF) range”, Materials Science And Engineering, vol. 111, pp. 113-123, 2004.
[2] A. F. L. Almeida, P. B. A. Fechini, L. C. Kretly, A. S. B. Sombra, "Ba$\mathrm{TiO}_{3}$ (BTO)- $\mathrm{CaCu}_{3} \mathrm{Ti}_{4} \mathrm{O}_{12}$ (CCTO) substrates for microwave devices and antennas”, Journal of Materials Science, vol. 41, number 14, pp. 4623-4631, July 2006.

[3] P. B. A. Fechini, A. Távora, L. C. Kretly, A. F. L. Almeida, M. R. P. Santos et all, "Microstrip antenna on a high dielectric constant substrate: $\mathrm{BaTiO}_{3}$ (BTO)-CaCu $\mathrm{Ti}_{4} \mathrm{O}_{12}$ (CCTO) composite screen-printed”, Journal of Electronic Materials, vol. 35, number 10, pp. 1848-1856, October 2006.

[4] www.cst.com

[5] C. A. Balanis, "Circular Cross-Section Waveguides and Cavities", in Advanced Engineering Electromagnetics, John Wiley and Sons, Inc., 1989.

[6] N. Kinayman e M. I. Aksun, "Microstrip Filters", in Modern Microwave Circuits, Artech House, Inc., 2005, pp. 415-428.

[7] G. L. Matthaei, L. Young, E. M. T. Jones, "Microwave Filters, Impedance-Matching Networks and Coupling Structures”, $2^{\text {nd }}$ edition, Artech House, Inc., 1980. 